The following scientific article was officially published in the Proceedings of the 11th International Conference on Information Sciences, Signal Processing and their Applications (ISSPA 2012), published by the IEEE. This article's citation is as follows:

Adankon, Mathias M., Jean Dansereau, Hubert Labelle, and Farida Cheriet. "Analysis of scoliosis trunk deformities using ICA." In 11th International Conference on Information Science, Signal Processing and their Applications (ISSPA), (2012): pp. 187-192.

doi: $\underline{10.1109 / \text { ISSPA.2012.6310543 }}$

The manuscript, as accepted by the publisher, is reproduced in the following pages.

(C) 2012 IEEE. Personal use of this material is permitted. Permission from IEEE must be obtained for all other users, including reprinting/republishing this material for advertising or promotional purposes, creating new collective works for resale or redistribution to servers or lists, or reuse of any copyrighted components of this work in other works. 


\title{
ANALYSIS OF SCOLIOSIS TRUNK DEFORMITIES USING ICA
}

\author{
Mathias M. Adankon, Jean Dansereau, Hubert Labelle and Farida Cheriet \\ Ecole Polytechnique de Montreal, Montreal, Canada \\ Sainte-Justine Hospital Research Center, Montreal, Canada
}

\begin{abstract}
This paper describes a method for analyzing scoliosis trunk deformities using Independent Component Analysis (ICA). Our hypothesis is that ICA can capture the scoliosis deformities visible on the trunk. Unlike Principal Component Analysis (PCA), ICA gives local shape variation and assumes that the data distribution is not normal. 3D torso images of 56 subjects including 28 patients with adolescent idiopathic scoliosis and 28 healthy subjects are analyzed using ICA. First, we remark that the independent components capture the local scoliosis deformities as the shoulder variation,the scapula asymmetry and the waist deformation. Second, we note that the different scoliosis curve types are characterized by different combinations of specific independent components.
\end{abstract}

\section{INTRODUCTION}

Independent Component Analysis (ICA) is a statistical method which attempts to express the observed data with a linear combination of mutually independent variables [8]. ICA finds the independent components, also called source, by maximizing the statistical independence of the estimated components. Many approaches are proposed in order to define independence, and present the basis of ICA algorithms. The important propositions can be divided into two main ideas:

1) Minimization of Mutual Information, where measures like Kullback-Leibler Divergence and maximum-entropy are used.

2) Maximization of non-Gaussianity, where kurtosis (fourthorder cumulant) and negentropy are used.

ICA was originally developed to deal with blind source separation problem. Since the recent increase of interest in ICA, various applications are designed based on this statistical technique. Among them, we have feature extraction or data representation, which consist of computing a small vector representation of data (sound, image, etc) $[11,18,4]$.

Mathias Adankon, Jean Dansereau and Farida Cheriet are with Ecole Polytechnique de Montreal, Montreal, Canada. (email: mathiasmahouzonsou.adankon@polymtl.ca,_ jean.dansereau@polymtl.ca, farida.cheriet@polymtl.ca) Hubert Labelle is with SainteJustine Hospital Research Center, Montreal, Canada (email: hubert.labelle@recherche-ste-justine.qc.ca). This work was supported by the GRSTB, the MENTOR program and the CIHR (Canadian Institutes of Health Research)
Recently, ICA is used for image segmentation task [17] and medical image analysis [6, 10, 21]. Boquete et al. [6] have proposed a thermographic image analysis based on ICA for automated detection of high tumor risk areas. Hassen et al [10] used ICA to built cardiovascular disease diagnosis based on magnetic resonance imaging. ICA in a high-dimensional space with sparse data was applied to landmarked 3D shapes resulting from the aortic segmentation. The aortic shape variations were captured by the independent components which are sorted using prior knowledge. The simple classification task in the 2D space spanned by the two first independent components was performed by a simple quadratic classifier. In [21], ICA is used to construct myocardial contraction shape analysis. A classification algorithm was built from the ICA components in order to automatically detect and localize abnormally contraction regions of the myocardium.

In this work, we propose to analyze 3D image of scoliosis trunk based on ICA in order to detect local scoliosis deformation on trunk. Adolescent idiopathic scoliosis (AIS) is a deformity of the spine manifested by asymmetry and deformities of the external surface of the trunk. It consists of a complex curvature in the three-dimensional space where the vertebrae rotation causes the distortion of the ribcage and the development of a hump on the back.

Human torso shape has many variations, and its analysis is very challenging. Usually, section decomposition method is used with cross section modeling $[14,1]$. The $3 \mathrm{D}$ image of the trunk is divided by cross section and each section is characterized by mathematical tools in order to extract some descriptors. Jaremko and al. $[15,16]$ proposed to compute from each cross-section the indexes describing back surface rotation, torso centroid line, principal axis orientation and half-area assymetry. In their studies, they show that these indexes are correlated to the internal deformity (spine). Cross-section decomposition is also used in [3] where each cross section is modeled as B-spline curve and dominant points are extracted to form the features vectors. Other technique used in the literature is orthogonal map. This technique consists to transform orthogonally the 3D image of the trunk by using certain $3 \mathrm{D}$ transform. In [2], the authors compute torso deformation indexes by using four transforms which are axial line, unfolded cylinder, enclosing cylinder and subtracting cylinder. Torso shape analysis is also performed by using machine learning techniques. Self-organizing Neural Networks (SNN) is used to parameterize the torso deformity [13]. 
However, many of previous techniques are developed in order to establish the relationship between the torso deformity and the spinal deformity, and the results are mitigated.

Our hypothesis is that scoliosis patients have external deformities which vary from important asymmetries of the trunk to local subtle deformations. A statistical analysis method like ICA will be used to detect and recognize local scoliosis deformations on the trunk. Also, the scoliosis trunk analysis proposed in this paper can be used to diagnosis scoliosis trunk by building a local probability density function for each Independent Component.

This paper is structured as follows. In Section 2, we describe the materials and the methodology used in this study. In Section 3, we present the experiments and results. We conclude this paper in Section 4.

\section{MATERIALS AND METHODS}

\subsection{Data acquisition}

Since many years, the acquisition of the trunk surface topography is part of the routine evaluation of scoliosis patients at Sainte-Justine University Hospital Center (SJUHC) in Montreal (Canada). The acquisition system is composed of four optical digitizers (CREAFORM, Montreal, Canada). Each optical digitizer contains one color CCD camera and a structured light projector. The acquisition process of each digitizer is as follows. Four fringe patterns, obtained by phase-shifting technique, are successively projected onto the surface. Based on the four resulting images and triangulation technique, the system computes the depth of each surface point relative to the reference plane. A fifth image, with no fringes, acquires the texture of the surface which is then mapped onto the $3 \mathrm{D}$ reconstruction.

For the reconstruction of the whole trunk, four scanners are placed around the patient (on the front, on the back and at $\pm 60^{\circ}$ laterally in front of the patient). Each digitizer reconstructs a portion of the trunk. During the acquisition, approximately 4 seconds, the patient stands still in the upright position with the arms slightly abducted in order not to obstruct the lateral scanners fields of view. Based on a multi-head calibration of the system that computes the rigid transformations between the digitizers, the 4 portions of the trunk are registered and merged using EM software. The resulting mesh is constituted of 40,000 to 70,000 nodes, depending on the size of the patient. The accuracy of this system was evaluated in [20], using markers placed on a mannequin whose coordinates were previously recorded by a computer measuring machine. The results showed a reconstruction accuracy of $1.4 \mathrm{~mm}$ over the whole torso and of $0.56 \mathrm{~mm}$ over the back.

\subsection{Features Extraction}

First of all, some pre-processing steps are performed before applying ICA, the training trunks have been aligned in order to remove unwanted variation. In this study, Generalized Procrustes alignment [9] is used for this task where a registration is done using isomorphic scaling, translation, and rotation.

Before alignment, each 3D image of scoliosis trunk is decomposed into 800 points ( 20 sections and 40 points per section) and the vector feature is built with all coordinates $(x, y, z)$ for each point. Thus, each torso is represented by one vector whose length is $2400=800 \times 3$.

In general, analyzing object based on statistical methods with an important number of features is not recommended because working in high-dimensional space involves the curse of dimensionality problem. For this purpose, PCA is used to reduce the dimensionality of the data. Figure 1 illustrate each step of the features extraction process from 3D image to features vector with 20 components.

\subsection{Dimensionality reduction}

In this study, PCA is used in order to reduce features dimensionality. PCA is a mathematical technique that uses an orthogonal transformation to convert a number of (possibly) correlated variables into a (smaller) number of uncorrelated variables. However, PCA focus on the components which provide a large variation and has not capacity to preserve the small variability. Thus, we select 20 components which provides the maximum of the total variance (99\%). Moreover, we verify the quality of the information contained in these components by testing the discrimination between the scoliosis curve type through cluster analysis.

Cluster analysis is performed using hierarchical clustering algorithm where the number of classes is not set in advance. After performing clustering analysis, a hierarchy of clusters was built with many levels, we show the dendrogram in Figure 2. The Distribution of the subjects among the classes is presented in Table 1 . We note that the selected principal components are efficient to contain the discriminative information of the different scoliosis curve type (thoracic major curve, lumbar major curves) and healthy subjects.

\subsection{ICA modeling}

Let us consider a dataset $\mathcal{D}$ comprising $\ell$ samples $\left\{x_{1}, \ldots, x_{\ell}\right\}$ with $x_{i} \in \mathbb{R}^{d}$. ICA attempts to find a linear transformation :

$$
X=A s
$$

where the statistical independence between the variables $s=\left(s_{1}, \ldots, s_{n}\right)$ is maximized. The random variables $s$ are called independent components (ICs), with $s_{i} \in \mathbb{R}^{d}$, and $A \in \mathbb{R}^{\ell \times n}$ is mixing matrix.

Thus, each sample $x_{i}$ of a dataset $\mathcal{D}$ is represented by a sum of the independent components weighted by the 


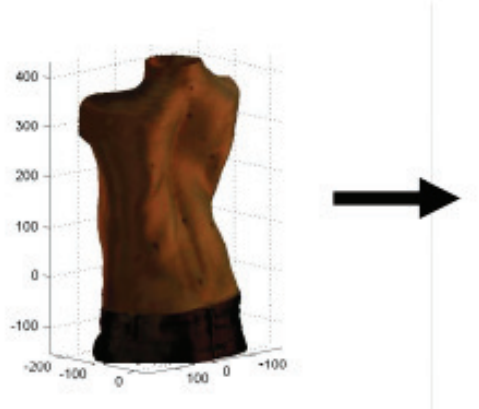

(a)

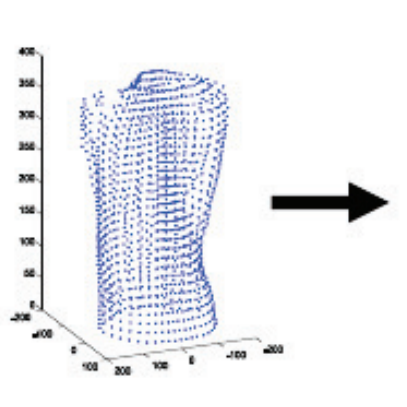

(b)

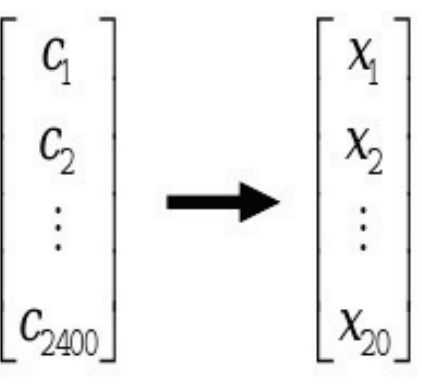

(c) (d)

Fig. 1. Features Extraction Process: (a) 3D Trunk image; (b) Cloud of 800 points; (c) Vector with 2400 components; (d) 20 Components resulting from PCA.

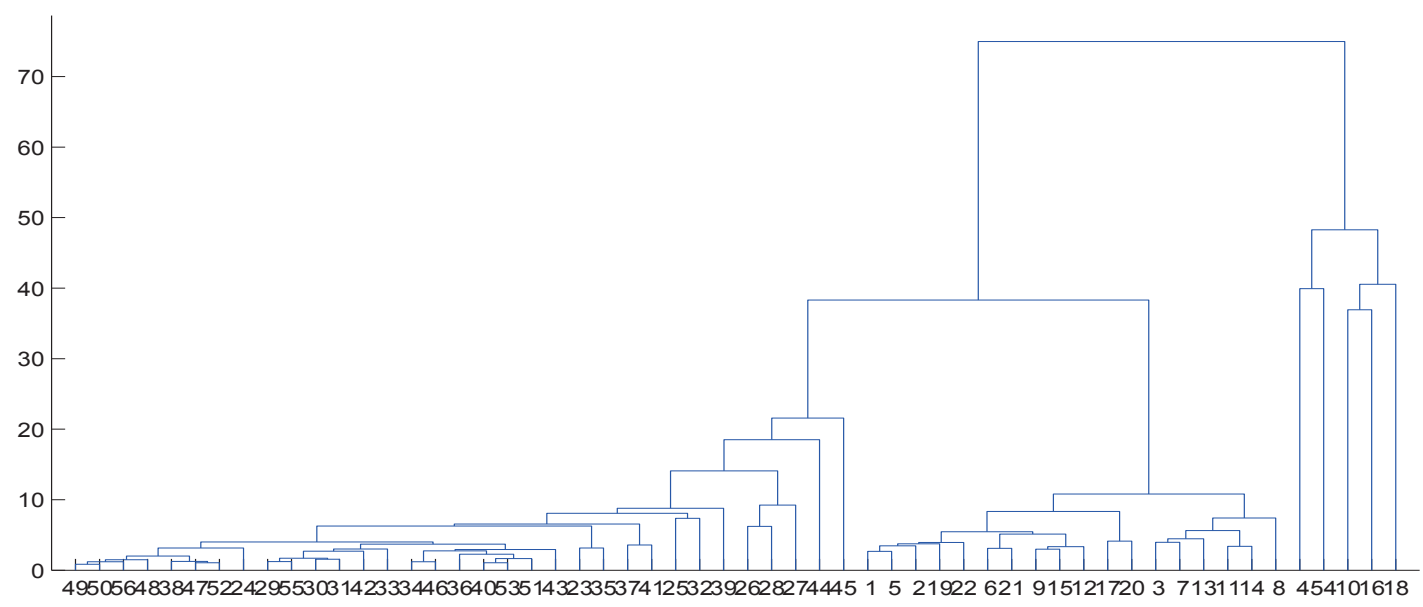

Fig. 2. Hierarchical clustering tree.

Table 1. Distribution of the patients among the classes found by hierarchical clustering analysis. One sample is viewed like outlier.

\begin{tabular}{lcc}
\hline Classes & Curve type & \# patients \\
\hline Class 1 & Lumbar major & $6 / 7$ \\
Class 2 & Thoracic major & $9 / 13$ \\
Class 3 & Thoracic major & $5 / 7$ \\
Class 4 & Healthy & $3 / 3$ \\
Class 5 & Lumbar major & $2 / 2$ \\
Class 6 & Healthy & $18 / 18$ \\
Class 7 & Healthy & $4 / 5$ \\
\hline
\end{tabular}

elements of the mixing matrix $A$ :

$$
x_{i}=\sum_{k=1}^{n} A_{i k} s_{k}
$$

The previous definition is a common classical defini- tion of ICA. However, it seems impossible to find a linear transformation that gives strictly independent components. Thus, in practice, some assumption is made on the data with a specific definition of the function that measures independence.

The following fundamental restrictions (in addition to the basic assumption of statistical independence) are imposed in order to assure the identifiability of the ICA model: a)All the independent components $s_{i}$ with the possible exception of one component, must be non-Gaussian.

b) The number of observed data $\ell$ must be at least as large as the number of independent components $n$, i.e., $\ell \geq n$. c)The matrix A must be of full column rank.

The statistical independence is the main key in all the algorithms design to perform ICA. Usually, an objective function for ICA is chosen and optimized. In the literature, we found various methods based on different objective function like measure of Nongaussianity (principle used by the FastICA [12]), Minimization of Mutual Infor- 
mation [5], Maximum Likelihood Estimation [7]. Maximum likelihood estimation is a very popular approach for estimating the ICA model. This technique is also connected to the infomax principle and it is shown in [19] that this method is essentially equivalent to minimization of mutual information.

ICA is applied to training data using the FastICA algorithm [12]. The independent components $s=\left(s_{1}, \ldots, s_{n}\right)$ and the projection of the training data (mixing matrix $A$ ) in the ICA space are estimated by maximizing the statistical independency. FastICA algorithm uses a fixed-point iteration technique which provides an accurate solution with a fast convergence. At each iteration, the algorithm finds one of all nongaussian independent components regardless of their probability distributions. It is 10-100 times faster than the other ICA algorithms which are based on conventional gradient descent method.

\section{EXPERIMENTS}

\subsection{Dataset and experimental setup}

The dataset consisted of 3D torso images of 56 subjects including 28 patients with adolescent idiopathic scoliosis (15 thoracic curves and 13 lumbar curves) and 28 healthy subjects who come to clinic because of their trunk appearance. The 3D trunk image and the radiography have been acquired at the same visit for each patient. The diagnosis of each individual is performed by an orthopedic surgeon from the X-ray images.

\subsection{Scoliotic trunk analysis}

We ran FastICA algorithm on the 56 samples represented by 20 features, we obtained 19 independent components $\left(s_{1}, s_{2}, \ldots, s_{19}\right)$.

ICA decomposes shapes into local shape descriptors [21]. In Figures 3, we plot ICA modes for pointing out the ICA capacity to capture local deformation. The $k-t h$ mode of trunk shape variation is defined by:

$$
z_{k}=\mu+s_{k} \delta
$$

where $\mu$ is the mean shape and $\delta=3 \sigma_{k}$ is three times the value of the variance of corresponding weight computed with the mixing matrix $A$.

$$
\sigma_{k}=\operatorname{Var}\left(A_{1 k}, A_{2 k}, \ldots, A_{\ell k}\right)
$$

In this study, we used three different representations of data corresponding to three spaces: shape space (cloud of points), PCA space (20 components) and ICA space (19 components). The projection through the three spaces are done according to the following equations:

$$
\begin{gathered}
X_{P C A}=\mu_{I C A}+X_{I C A} * S \\
X_{\text {Shape }}=\mu_{P C A}+X_{P C A} * V
\end{gathered}
$$

where

$S$ is the matrix of the independent components; $V$ is the matrix of the principal components;
$\mu_{I C A}$ is the mean vector of ICA model; $\mu_{P C A}$ is the mean vector of PCA model;

$X_{\text {Shape }}=\left(x_{1}, y_{1}, z_{1}, x_{2}, y_{2}, z_{2}, \ldots, x_{800}, y_{800}, z_{800}\right)$.

The local deformation captured by each IC is coded in color (the mean shape is in blue and the deformation is in red) with respect to the mean shape value. Figure 3 shows different IC which capture the shoulder variation,the scapula asymmetry and the waist deformation. Also, the prototypes trunk that illustrate each local deformation captured by the ICs are shown. These prototypes are selected by using the mode of the corresponding weight contained in the mixing matrix.

\subsection{Analysis of scoliosis curve type with respect to the independent components}

In this section, we analyzed the behavior of each independent component according to scoliosis curve type. The mean value of each IC weight is computed with respect to the scoliosis curve type (thoracic and lumbar curves). The results are shown on Figure 4. We note that among the nineteen components, eleven components (IC\#2, 3, 5, $6,7,8,11,12,13,14,15)$ are identified as capturing the deformation of the trunk of the patients who have thoracic curve and ten components (IC\#3, 4, 7, 9, 10, 12, 13, 17, $18,19)$ for the deformation of the trunk of the patients who have lumbar curve. Considering the significance of the components, the IC\#11 which corresponds to the scapula deformation have a high weight for thoracic curve. In fact, thoracic curve causes often the ribcage distortion which produces the scapula deformation.

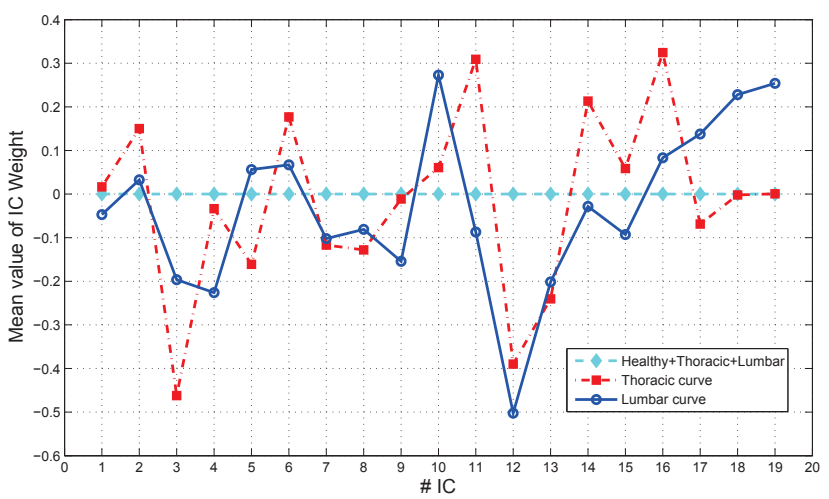

Fig. 4. Comparison of the weight of each independent component w.r.t. scoliosis curve type.

\section{CONCLUSION}

Scoliosis is a three-dimensional deformity of the spine and the ribcage. This deformity affects the torso and causes an apparent deformation of the external trunk surface. The $3 \mathrm{D}$ trunk image is constituted of a 55,000 points in average. Thus, the trunk image analysis using directly the row data is a challenging task and the design of an efficient analysis framework could be useful. 

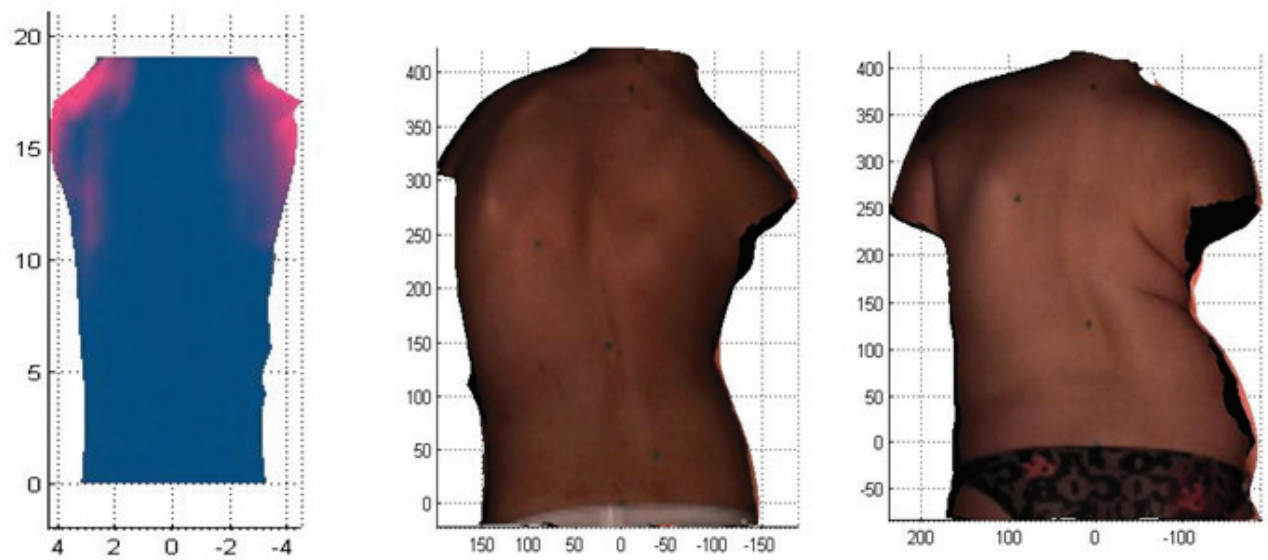

\section{(a) Shoulders asymmetry}
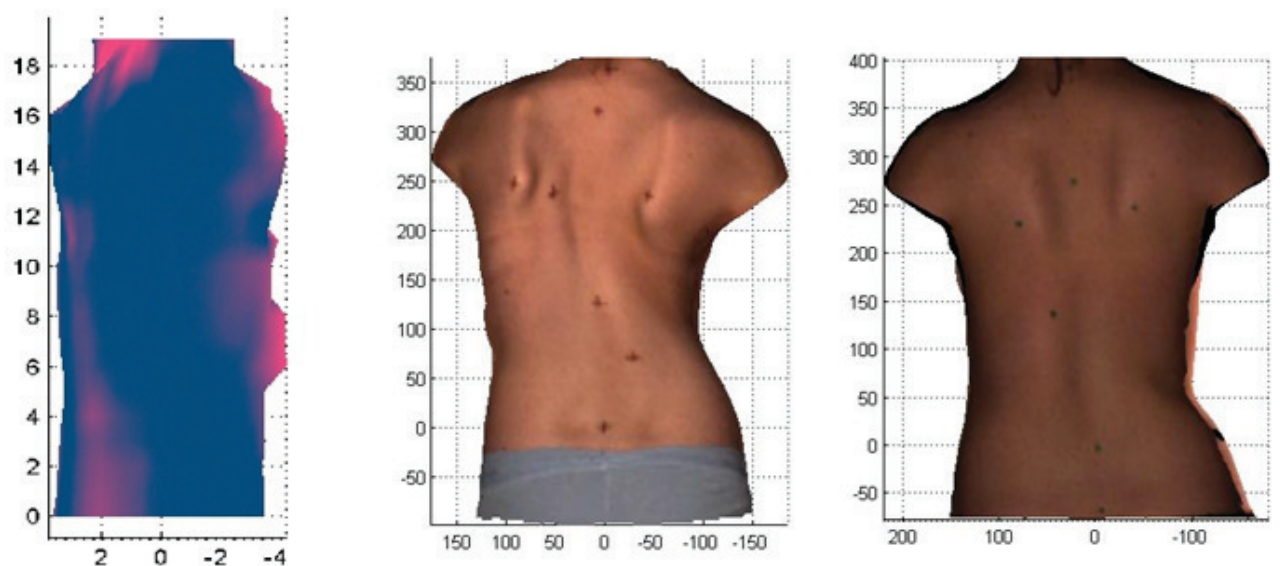

(b) Left side (waist) deformity
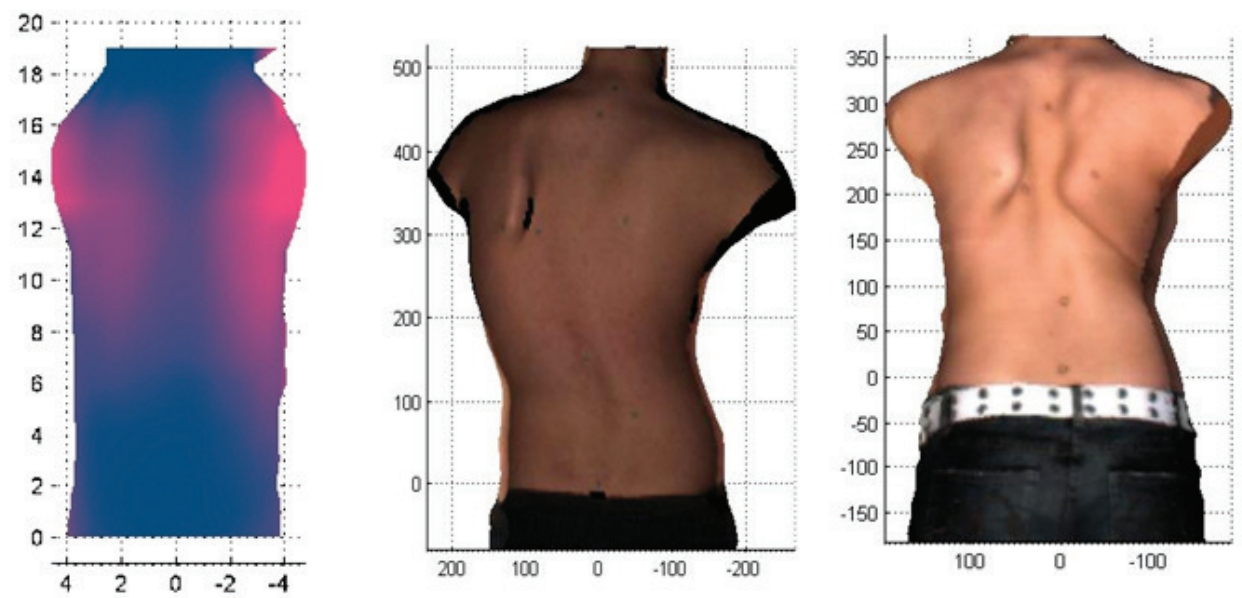

\section{(c) Scapula deformity}

Fig. 3. Illustration of three independent components which capture the shoulder asymmetry,the scapula deformity and the waist deformation.

In this work, trunk analysis is performed based on ICA which has the capacity to capture the local deformity. Unlike PCA, which gives a global shape variation (principal direction of the data), ICA can be exploited to detect lo- cal shape deformities. We find that the local scoliosis deformities as the shoulder variation,the scapula asymmetry and the waist deformation are captured by the independent components. 
Concerning scoliosis diagnosis, it will be interesting to select the dominant independent components which capture the scoliosis deformities. Then, the follow up will be performed by analysing the variation of the weight of these components based on the assumption that they are independent statistically. Also in future work, the study will be extended to the double major curve which analysis is more complex than the simple curve like thoracic or lumbar curve.

\section{References}

[1] Mathias M. Adankon, Hubert Labelle, Jean Dansereau, and Farida Cheriet. Scoliosis curve type classification from $3 \mathrm{~d}$ trunk image using kernel machine. In SPIE Medical Imaging, 2012.

[2] P.O. Ajemba, N.G. Durdle, D.L. Hill, and V.J. Raso. Classifying torso deformity in scoliosis using orthogonal maps of the torso. Medical and Biological Engineering and Computing, 45(6):575-584, june 2007.

[3] P.O. Ajemba, N.G. Durdle, and V.J. Raso. Characterizing torso shape deformity in scoliosis using structured splines models. Biomedical Engineering, IEEE Transactions on, 56(6):1652 -1662, june 2009.

[4] K. Bae, S. Noh, and J. Kim. Iris feature extraction using independent component analysis. In Audioand Video-Based Biometric Person Authentication, pages 1059-1060. Springer, 2003.

[5] A.J. Bell and T.J. Sejnowski. An informationmaximization approach to blind separation and blind deconvolution. Neural computation, 7(6):11291159, 1995.

[6] L. Boquete, S. Ortega, J.M. Miguel-Jiménez, J.M. Rodríguez-Ascariz, and R. Blanco. Automated detection of breast cancer in thermal infrared images, based on independent component analysis. Journal of Medical Systems, pages 1-9, 2010.

[7] J.F. Cardoso. High-order contrasts for independent component analysis. Neural computation, 11(1):157-192, 1999.

[8] P Comon. Independent component analysis, a new concept ? Signal Processing, Elsevier, 36(3):287314, 1994.

[9] J.C. Gower. Generalized procrustes analysis. Psychometrika, 40(1):33-51, 1975.

[10] Michael Hansen, Fei Zhao, Honghai Zhang, Nicholas Walker, Andreas Wahle, Thomas Scholz, and Milan Sonka. Detection of connective tissue disorders from $3 \mathrm{~d}$ aortic mr images using independent component analysis. In Reinhard Beichel and Milan Sonka, editors, Computer Vision Approaches to Medical Image Analysis, volume 4241 of Lecture
Notes in Computer Science, pages 13-24. Springer Berlin / Heidelberg, 2006.

[11] P.O. Hoyer and A. Hyvärinen. Independent component analysis applied to feature extraction from colour and stereo images. Network: Computation in Neural Systems, 11(3):191-210, 2000.

[12] A. Hyvarinen, J. Karhunen, and E. Oja. Independent component analysis, volume 26 . Wiley-Interscience, 2001.

[13] P. Igwe, M. Emrani, S. Adeeb, and D. Hill. Assessing torso deformity in scoliosis using self-organizing neural networks (snn). In Machine Learning and Applications, 2008. ICMLA '08. Seventh International Conference on, pages 497 -502, dec. 2008.

[14] J. L. Jaremko. Estimation of Scoliosis Severity from the Torso Surface by Neural Networks. Ph.D. Thesis, Dept. Biomed. Eng., Univ. Calgary, 2003.

[15] J L Jaremko, P Poncet, J Ronsky, J Harder, J Dansereau, H Labelle, and R F Zernicke. Estimation of spinal deformity in scoliosis from torso surface cross sections. Spine (Phila Pa 1976), 26(14):1583-91, 2001.

[16] Jacob L. Jaremko, Philippe Poncet, Janet Ronsky, James Harder, Jean Dansereau, Hubert Labelle, and Ronald F. Zernicke. Indices of torso asymmetry related to spinal deformity in scoliosis. Clinical Biomechanics, 17(8):559 - 568, 2002.

[17] J. Koikkalainen and J. Lotjonen. Image segmentation with the combination of the pca- and ica-based modes of shape variation. In Biomedical Imaging: Nano to Macro, 2004. IEEE International Symposium on, pages 149 - 152 Vol. 1, april 2004.

[18] J.H. Lee, H.Y. Jung, T.W. Lee, and S.Y. Lee. Speech feature extraction using independent component analysis. In Acoustics, Speech, and Signal Processing, 2000. ICASSP'00. Proceedings. 2000 IEEE International Conference on, volume 3, pages 16311634. Ieee, 2000.

[19] E. Oja and A. Hyvarinen. Independent component analysis: algorithms and applications. Neural Network, 13:411-430, 2000.

[20] V. Pazos, F. Cheriet, L. Song, H. Labelle, and J. Dansereau. Accuracy assessment of human trunk surface $3 \mathrm{~d}$ reconstructions from an optical digitising system. Medical and Biological Engineering and Computing, 43(1):11-15, 2005.

[21] A. Suinesiaputra, A.F. Frangi, T. Kaandorp, H.J. Lamb, J.J. Bax, J. Reiber, and B. Lelieveldt. Automated detection of regional wall motion abnormalities based on a statistical model applied to multislice short-axis cardiac mr images. Medical Imaging, IEEE Transactions on, 28(4):595 -607, april 2009. 